\title{
Current Shot Noise and Bunching of Electrons in Multilevel Quantum Dots
}

\author{
G. MichąeK And B.R. BuŁKa \\ Institute of Molecular Physics, Polish Academy of Sciences \\ Smoluchowskiego 17, 60-179 Poznań, Poland
}

\begin{abstract}
Currents and their fluctuations in multilevel quantum dots are studied theoretically in the limit of sequential tunneling. The spin degrees of freedom, many-body electronic states (singlet and triplet) as well as relaxation processes between the levels of the quantum dots are considered. In general, due to the rapid relaxation processes the shot noise is sub-Poissonian, however for a large polarization of the outgoing currents from the singlet and triplet states one gets the super-Poissonian type of the shot noise due to the bunching of tunneling events.
\end{abstract}

PACS numbers: 73.23.--b, 72.70.+m

\section{Introduction}

Small semiconducting or metallic quantum dots are seen as potential candidates for building of novel electronic devices because of the small size and low energy dissipation. The multilevel quantum dots (QDs) are very interesting because many-body electronic states (e.g. singlet and triplet) can be use as qubits in quantum computations, see e.g. [1]. The fundamental problems appearing in connection with quantum computations are decoherence and dissipation of energy, which destroy quantum entanglement. The energy relaxation processes from excited states can be induced by interaction of electrons on the QD with phonons bath. Such transitions are very fast and accompanied by acoustic phonon emission [2]. In contrast, if the transition involves spin-flip processes, on the QDs there appear long-lived (more than few $\mu \mathrm{s}$ ) spin states, which can cause spin blockade phenomena $[2,3]$.

In the nanoscale devices out of equilibrium, current fluctuations provide a great deal of insight into the relevant transport mechanisms, correlations and different relaxation processes [4].

\section{Model and method of calculations}

The system under consideration is presented schematically in Fig. 1a. We assume that the gate voltage shifted the levels down and two electrons can oc- 
cupy the quantum dot. Let the singlet state $(s)$ be a lower energy state than the triplet one $(t)$ (as it occurs in most situations), and the source-drain voltage is high enough, so the singlet and the triplet states lie in the voltage window and both participate in electronic transport (see Fig. 1a). Two single-electrons levels are below the chemical potentials of the electrodes, and one electron (with the spin $\sigma=\uparrow$ or $\downarrow$ ), which occupies the level $n=1$ or $n=2$, is always present at the QD. An electron (with the spin $\sigma=\uparrow$ or $\downarrow$ ) can tunnel (with the transfer rate $\gamma_{\mathrm{L} n s}$ or $\left.\gamma_{\mathrm{L} n t}, n=1,2\right)$ from the left electrode to the empty state. An electron can leave the singlet (triplet) state $|s\rangle(|t\rangle)$ and tunnel to the right electrode with the transfer rate $\gamma_{\mathrm{R} n s}\left(\gamma_{\mathrm{R} n t}\right)$. A relatively large source-drain voltage is chosen in order to eliminate backward tunneling processes, which would degrade the relaxation time measurements [2]. All tunneling rates $\gamma_{\mathrm{L}(\mathrm{R}) n s(t)}$ are assumed to be small $\hbar \gamma_{\mathrm{L}(\mathrm{R}) n s(t)} \ll k_{\mathrm{B}} T$. It implies that the tunnel resistances of the left and right barriers are much larger than the quantum resistance $R_{Q}=h / 2 e^{2}$ and electronic transport can be described within the sequential tunneling approach [5]. We include also relaxation processes with the transfer rate $w_{t}$ and $w_{21}$ for transition from the triplet to the singlet state and from the $n=2$ to the $n=1$ state, respectively (Fig. 1a). It is assumed that the temperature is low $\left(k_{\mathrm{B}} T \ll w_{t}, \hbar w_{21}\right)$ and excitations from the lower to the higher state are absent.
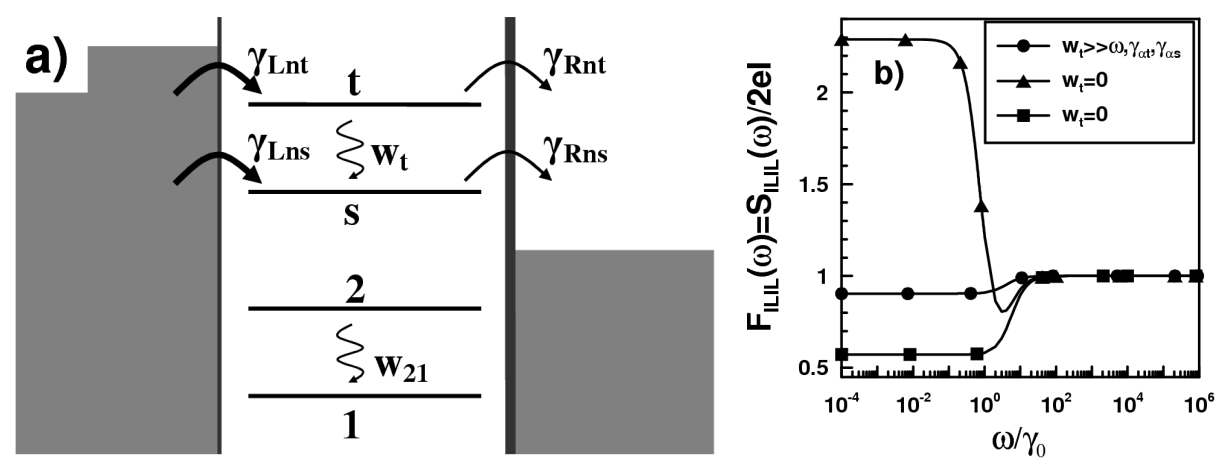

Fig. 1. (a) Schematic view of the system. (b) Fano factor of the current noise as a function of the frequency $\omega / \gamma_{0}$. The parameters are: $\gamma_{\mathrm{L} t}=1.5 \gamma_{0}, \gamma_{\mathrm{L} s}=3 \gamma_{0}$, $\gamma_{\mathrm{R} t}=\gamma_{0}, \gamma_{\mathrm{R} s}=0.1 \gamma_{0}, w_{21}=0, e=1$ (for a large relaxation rate $w_{t}$ - circles, and for $w_{t}=0$ - triangles), while $\gamma_{\mathrm{R} s}=0.8 \gamma_{0}$ (for $w_{t}=0$ - square). $\gamma_{0}$ is taken as unity in our calculations.

Electronic transport is governed by the master equation $\mathrm{d} \hat{p}(N) / \mathrm{d} t=\hat{M} \hat{p}(N)$, where $\hat{p}(N)=\left\{p_{1 \sigma}, p_{2 \sigma}, p_{s}, p_{t S}\right\}(\sigma=\{\uparrow, \downarrow\}, S=\{\overline{1}, 0,1\})$ denotes the probability to find an electron at one of the levels: 1,2 , singlet, triplet. In the tunnel matrix $\hat{M}$ there are included all transition processes described above.

Fluctuations in the system are studied within the generation-recombination approach for multi-electron channels [6, 7]. The Fourier transform of the correla- 
tion function of the quantity $X$ can be expressed as $[6,7]$ :

$$
S_{X X}(\omega)=4 \sum_{N, N^{\prime}} X(N) \operatorname{Re}\left(G_{N, N^{\prime}}(\omega)\right) X\left(N^{\prime}\right) p\left(N^{\prime}\right)
$$

where the elements of the Green function $G_{N, N^{\prime}}(\omega) \equiv[i \omega \hat{1}-\hat{M}]_{N N^{\prime}}^{-1}, N\left(N^{\prime}\right)$ indexes final (initial) states. The correlation function between the currents $I_{\alpha}$ and $I_{\beta}$ in the tunnel junctions $\alpha$ and $\beta$ can be expressed by formula

$$
S_{I_{\alpha} I_{\beta}}(\omega)=\delta_{\alpha \beta} S_{\alpha}^{\mathrm{Sch}}+S_{I_{\alpha} I_{\beta}}^{\mathrm{c}}(\omega)
$$

where $S_{\alpha}^{\text {Sch }}=2 e I$ is the high frequency limit $(\omega \rightarrow \infty)$ of the shot noise (Schottky noise), and the second part is frequency dependent [7].

\section{Results and conclusions}

In order to show relevance of the relaxation processes $w_{t}$ we consider asymmetric barriers $\gamma_{\mathrm{L} n s(t)}>\gamma_{\mathrm{R} n s(t)}$. We also assume that the transfer rates to the states $n=1,2$ are equal, i.e. $\gamma_{\alpha 1 t}=\gamma_{\alpha 2 t}=\gamma_{\alpha t}$ and $\gamma_{\alpha 1 s}=\gamma_{\alpha 2 s}=\gamma_{\alpha s} / 2$, but $\gamma_{\alpha t} \neq \gamma_{\alpha s}$. We found that the stationary current $I$ is independent of the transfer rate $w_{21}$ between the single-electron states.

For the rapid relaxation processes from the triplet to the singlet state $\left(w_{t} \gg \omega, \gamma_{\alpha s(t)}\right)$ both the singlet and the triplet state contribute to the incoming current (through the left junction), while only singlet state contributes to the outgoing current (through the right junction), because outgoing current from the triplet state vanishes. We found that the Fano factor

$$
F_{I L I L}(\omega) \equiv \frac{S_{I L I L}(\omega)}{2 e I}=1-\frac{2 \gamma_{\mathrm{R} s}\left(\gamma_{\mathrm{L} s}+3 \gamma_{\mathrm{L} t}\right)}{\omega^{2}+\left(\gamma_{\mathrm{L} s}+3 \gamma_{\mathrm{L} t}+4 \gamma_{\mathrm{R} s}\right)^{2} / 4}
$$

is always less than 1 , for $\omega=0$ (see Fig. 1b) (circles). It means that the rapid relaxation processes (caused e.g. by electron-phonon interactions [2]) leads to the sub-Poissonian type of the shot noise.

In the opposite case, when the triplet-singlet relaxation is slow (e.g. involve spin-flip [2]) or even without dissipation (for $w_{t}=0$ ) the low-frequency shot-noise can be both sub- or super-Poissonian (as one can see in Fig. 1b (sub-: squares, super-: triangles)). We calculated the Fano factor for $\omega=0$ :

$$
\begin{aligned}
& F_{I L I L}(\omega=0) \\
& \quad=1-\frac{8 \gamma_{\mathrm{R} s} \gamma_{\mathrm{R} t}\left(\gamma_{\mathrm{L} s} \gamma_{\mathrm{R} t}+3 \gamma_{\mathrm{L} t} \gamma_{\mathrm{R} s}\right)-6 \gamma_{\mathrm{L} s} \gamma_{\mathrm{L} t}\left(\gamma_{\mathrm{R} s}-\gamma_{\mathrm{R} t}\right)^{2}}{\left(\gamma_{\mathrm{L} s} \gamma_{\mathrm{R} t}+3 \gamma_{\mathrm{L} t} \gamma_{\mathrm{R} s}+4 \gamma_{\mathrm{R} s} \gamma_{\mathrm{R} t}\right)^{2}} .
\end{aligned}
$$

From this formula one can find that the low-frequency super-Poissonian current noise appears when the transfer rates fulfill the following condition: $\left(\gamma_{\mathrm{R} s}-\gamma_{\mathrm{R} t}\right)^{2} / 4 \gamma_{\mathrm{R} s} \gamma_{\mathrm{R} t}>\gamma_{\mathrm{R} s} / \gamma_{\mathrm{L} s}+3 \gamma_{\mathrm{R} t} / \gamma_{\mathrm{L} t}$. It implies that the enhancement of the current noise occurs for a large polarization of the outgoing currents from the singlet and the triplet states. In the case, dynamical channel blockade is formed: the electrons from the singlet state leave the dot with the rate $\gamma_{\mathrm{Rs}}=0.1 \gamma_{0}$ much smaller than the rate $\gamma_{\mathrm{R} t}$ for electrons from the triplet state. As a consequence 
an effective bunching of tunneling events appears, leading to super-Poissonian low-frequency noise $[8,9]$. However, for higher frequencies, the anticorrelations between currents occurs $\left(S_{I L I L}^{\mathrm{c}}(\omega)<0\right)$, and current noise is reduced below Poissonian value, even if the system is in the regime of dynamical channel blockade, as is clearly seen in Fig. 1b (triangle).

\section{Acknowledgments}

The work was supported in part by the Ministry of Science and Higher Education within a research project for years 2004-2007, as part of the European Science Foundation EUROCORES Programme FoNE by funds from the Ministry of Science and Higher Education and EC 6FP (contract No. ERAS-CT-2003980409), and the EC project RTNNANO (contract No. MRTN-CT-2003-504574).

\section{References}

[1] S. Das Sarmaa, R. de Sousab, X. Huc, B. Koillerd, Solid State Commun. 133, 737 (2005).

[2] T. Fujisawa, D.G. Austing, Y. Tokura, Y. Hirayama, S. Tarucha, J. Phys., Condens. Matter 15, R1395 (2003).

[3] D. Weinmann, W. Häusler, B. Kramer, Phys. Rev. Lett. 74, 984 (1995).

[4] Ya.M. Blanter, M. Büttiker, Phys. Rep. 336, 1 (2000).

[5] G. Schön, in: Quantum Transport and Dissipation, Eds. T. Dittrich, P. Hänggi, G.-L. Ingold, B. Kramer, G. Schön, W. Zwerger, Wiley-VCH Verlag, New York 1998, p. 149.

[6] K.M. van Vliet, J.R. Faset, in: Fluctuation Phenomena in Solids, Ed. R.E. Burgess, Academic Press, New York 1965, p. 267.

[7] A.N. Korotkov, Phys. Rev. B 49, 10381 (1994).

[8] S. Gustavsson, R. Leturcq, B. Simovič, R. Schleser, P. Studerus, T. Ihn, K. Ensslin, Phys. Rev. B 74, 195305 (2006).

[9] W. Belzig, Phys. Rev. B 71, 161301 (2005). 